Benchmarks

\title{
Simplified and versatile method for isolation of high-quality RNA from
} pancreas

\author{
Michelle Griffin ${ }^{1 *}$, Maisam Abu-El-Haija ${ }^{1 *}$, Marwa Abu-El-Haija ${ }^{1}$, \\ Tatiana Rokhlina ${ }^{2}$, and Aliye $\mathrm{Uc}^{1}$ \\ ${ }^{1}$ Departments of Pediatrics and ${ }^{2}$ Internal Medicine, University of Iowa \\ Carver College of Medicine, Iowa City, IA, USA
}

BioTechniques 52:332-334 (May 2012) doi 10.2144/0000113862

Keywords: pancreas; RNA; PCR; microarray

${ }^{*}$ M.G. and M.A.-E.-H. contributed equally to this work.

Isolation of high-quality RNA from pancreas is challenging because the organ contains large quantities of RNases and undergoes autolysis upon harvest. Here we present a simplified perfusion method of the pancreas using an RNase inhibitor. The technique consistently yields high-quality RNA from stored pancreas samples suitable for molecular biology applications, including quantitative RT-PCR. Yields are comparable to RNA isolated from pancreas immediately, but superior to RNA isolated from stored samples that were snap-frozen or immersed in an RNase inhibitor solution. In addition, when compared to the previously reported in situ ductal perfusion technique, our method does not cause histological artifacts.

Obtaining high-quality and intact RNA from the pancreas is a crucial starting point for many molecular biology applications. This has proven to be difficult, because pancreas contains large quantities of proteases, DNases and RNases that initiate an autolytic process almost immediately upon harvest. Despite several approaches, including rapid removal of pancreas from the abdominal cavity, homogenizing at cold temperatures, inhibition of contaminating RNases, enrichment of mRNA species, and elimination of contaminating DNA (1-7), isolation of intact and high-quality RNA from pancreas remains a challenge. Here we report a method that consistently yields high quality RNA from pancreas and permits other applications, including tissue histopathology. Although similar to the in situ ductal perfusion technique reported by Mullin et al. (3), our method is simpler, does not require cannulation of the pancreatic duct, and does not cause histological artifacts.

All experimental animal protocols were approved by the University of Iowa Institutional Animal Care and Use Committee. Newborn piglets ( $<24 \mathrm{~h}$ of age) (Sus scrofa) and adult mice were euthanized, and pancreas was harvested within $10 \mathrm{~min}$ following euthanasia. The pancreas was dissected in a half, with one half acting as control for the conventional sample preparation and the other half perfused with the RNase inhibitor solution RNAlater (Qiagen, Valencia, CA, USA) ( $n=6$ for piglets, $n=$ 5 for mice). Results were expressed as mean \pm SEM, and statistical analysis was done by one-way analysis of variance (ANOVA) with Dunnett's Multiple Comparison Post-Test.
One-half of the pancreas was cut into small pieces, placed in cryovials, and snap-frozen in liquid nitrogen, or cut into small pieces and placed in RNAlater and kept overnight at $4^{\circ} \mathrm{C}$, or processed immediately for RNA isolation after brief storage in RNAlater. Long-term storage was at $-80^{\circ} \mathrm{C}$.

The other half of the pancreas was perfused with RNAlater using a $1-\mathrm{mL}$ syringe and a 26 gauge $\times 1 / 2$ inch needle. Multiple injections of the pancreas were made until a visible swelling was observed (Figure 1). The pancreas perfused with RNAlater was then cut into small pieces, placed in cryovials, snap-frozen in liquid nitrogen, and stored at $-80^{\circ} \mathrm{C}$.

Tissue homogenization was performed using Fisherbrand Disposable Pestle System (no. 03-392-106 grinder, 0.5-mL pestle size; Fisher Scientific, Pittsburgh, PA, USA), and RNA was extracted using Qiagen RNeasy Lipid Tissue Minikit (no.74804; Qiagen). The optional DNase digestion step was performed to prevent genomic DNA contamination.

Following RNA extraction, RNA integrity numbers (RIN) were obtained using Agilent 2100 bioanalyzer system (Agilent Technologies, Santa Clara, CA, USA). This system determines the integrity of RNA and automatically calculates RNA concentration in relation to the relative ratio of $18 S$ and 28 S ribosomal peaks (8). The RIN software uses a numbering system from 1 to 10 , with 1 being the most degraded profile, and 10 being the most intact RNA.

RNA was degraded with decreased $18 \mathrm{~S}$ and $28 \mathrm{~S}$ ribosomal RNA bands and increased baseline signal prior to $18 \mathrm{~S}$ and $28 \mathrm{~S}$ bands in piglet pancreata that were snap-frozen or kept at $4^{\circ} \mathrm{C}$ overnight following immersion into RNAlater after the tissue harvest (Figure 2, $\mathrm{A}, \mathrm{B}, \mathrm{E}$, and $\mathrm{F}$ ). RNA isolated from pancreas either immediately or after perfusion with RNAlater yielded the best results (Figure 2, C, D-F). Perfusing pancreas with RNAlater was superior to immersing in RNAlater, presumably because it allowed RNAlater to penetrate into the organ and stop the degradation process faster. The low yield of RNA from the immediately frozen pancreas samples was consistent with a rapid degra-

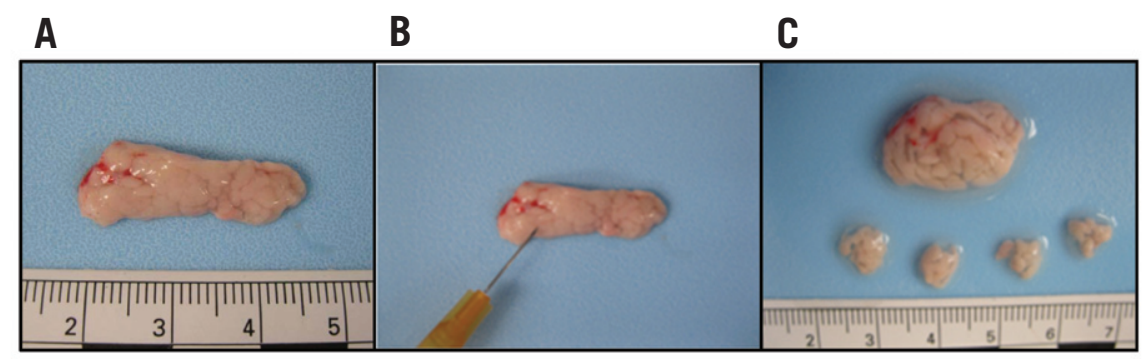

Figure 1. Pancreas perfusion technique. (A) Newborn pig pancreas before perfusion. (B) The perfusion of RNAlater into newborn porcine pancreas using a 1 -mL syringe and a 26 gauge $\times 1 / 2$ inch needle. (C) Swollen pancreas following RNAlater injections (top), and pancreas cut into small pieces after injections (bottom). 
dation process initiated by proteases, DNases, and RNases in the pancreas (9).

The quantity of RNA isolated with our pancreas perfusion technique was comparable to other methods in newborn pigs [immediate, $153 \pm 19 \mathrm{ng} / \mu \mathrm{L}$; perfusion, $115 \pm 17 \mathrm{ng} / \mu \mathrm{L}$; immersion, $143 \pm 25 \mathrm{ng} / \mu \mathrm{L}$; snap-freeze, 200 $\pm 23 \mathrm{ng} / \mu \mathrm{L} ; P$, nonsignificant (ns) between groups]. In adult mice, the quantity of RNA was highest with immediate RNA isolation and pancreas perfusion techniques and lowest with immersing pancreas in RNAlater or snap-freezing [immediate, $1357 \pm 353 \mathrm{ng}$ / $\mu \mathrm{L}$; perfusion, $697 \pm 203 \mathrm{ng} / \mu \mathrm{L}$; immersion, $547 \pm 155 \mathrm{ng} / \mu \mathrm{L}$; snap-freeze, $496 \pm 133 \mathrm{ng} /$ $\mu \mathrm{L} ; P$, ns for immediate versus perfusion; $P<$ 0.05 for immediate versus immersion, and for immediate versus snap-freeze].

To determine whether RNA isolated with perfusion of the pancreas could be used for downstream molecular biology applications, we performed quantitative RT-PCR (qPCR) for cystic fibrosis transmembrane conductance regulator (CFTR) using a methodology we previously described (10). RNA isolated with our method resulted in excellent amplification (Figure 3).

Perfusing pancreas with an RNase inhibitor has the potential to disrupt the tissue structure and create artifacts (John Alan Kiernan, personal communication) (11). For an optimal preservation of the tissue structure, a section of the pancreas should be removed first for histological examination, followed by RNAlater perfusion for RNA extraction (11). To test whether the previously described duct perfusion method (3) disrupts tissue architecture, we examined histological sections of pancreas after perfusion with RNAlater via the pancreatic duct and compared with the sections that were not subjected to duct perfusion. Duct perfusion with RNAlater disrupted the architecture of the pancreas (Figure 4). We next tested whether the in situ duct perfusion technique would be feasible for histopathological studies if an adequate sample $(\sim 5 \mathrm{~mm})$ was removed from adult mouse pancreas before the perfusion. Removal led to the leakage of RNAlater into the abdominal cavity, preventing the visible ballooning and translucency of the pancreas described with this method $(n=3)$ (3). Therefore, in contrast to Mullin's technique, our method offers the versatility of performing molecular biology applications, as well as tissue histopathology.

We used RNAlater, a commercially available RNase inhibitor. Because the ingredients of RNAlater are not available, we cannot directly compare the results to other RNA isolation agents, including reagents containing phenol and guanidine thiocyanate to inhibit RNase activity (i.e., TRI-reagent, TRIzol).
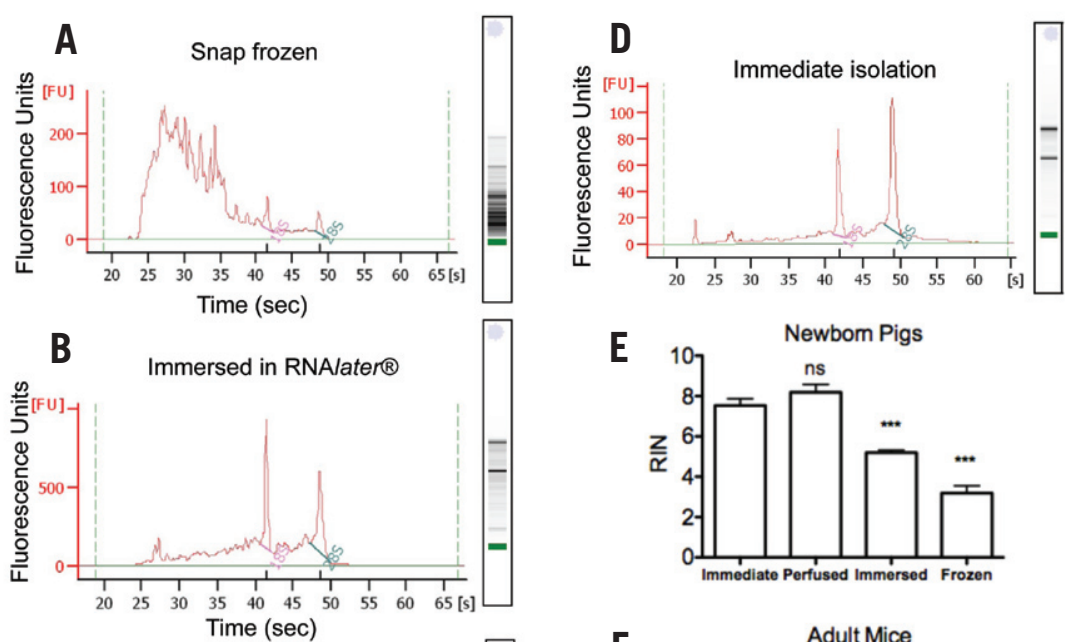

E Newbom Pigs
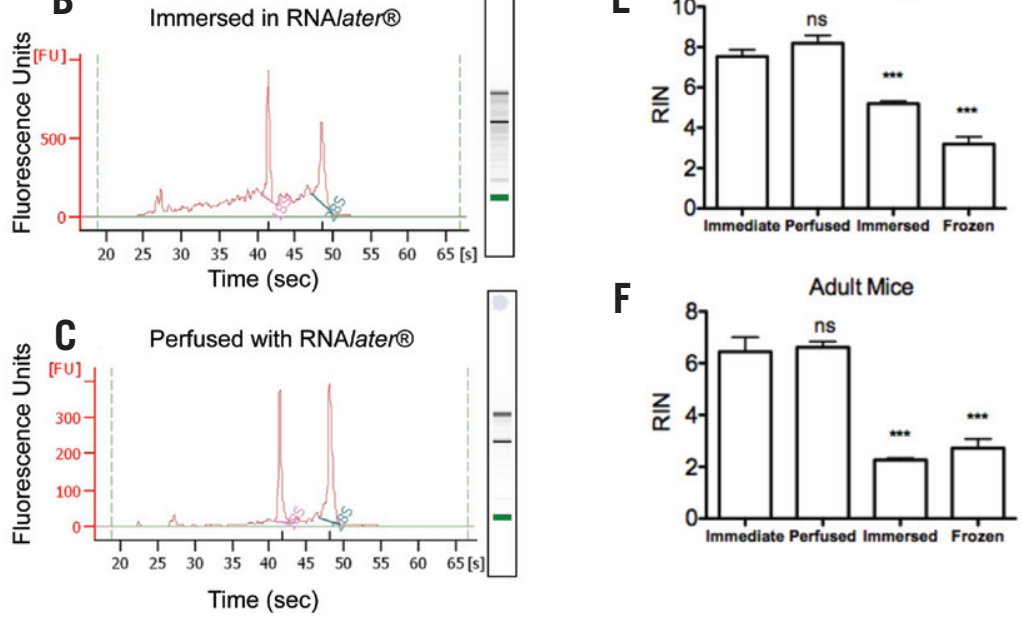

Figure 2. Pancreas perfusion yields high-quality RNA. Electropherograms and electrophoretic tracings from the piglet pancreas samples that were snap-frozen (A), immersed in RNAlater (B), perfused with RNAlater (C). (A and B) Snap-frozen samples or samples immersed in RNAlater showed degradation of RNA with decreased 18 S and 28S ribosomal RNA bands and increased baseline signal prior to the $18 \mathrm{~S}$ and 28 bands. Electrophoretic trace shows multiple bands that correspond to short fragments of RNA, consistent with degradation. Pancreata that were perfused with RNAlater (C) or immediate RNA isolation (D) gave high-quality RNA, with no increased baseline signal prior to $18 \mathrm{~S}$ and 28 bands or increased number of bands in the electrophoretic trace. Summary of RINs from RNA isolated from newborn pig (E) and adult mouse pancreas (F) immediately (immediate), after perfusion with RNAlater (perfused), immersion in RNAlater (immersed), or after snap-freeze (frozen) (mean \pm SEM). $N=6$ for newborn pig and $N=5$ for adult mouse pancreas. $P$ values are shown (ns, compared with RNA isolated immediately; ${ }^{* * *} P<0.001$ compared with RNA isolated immediately).

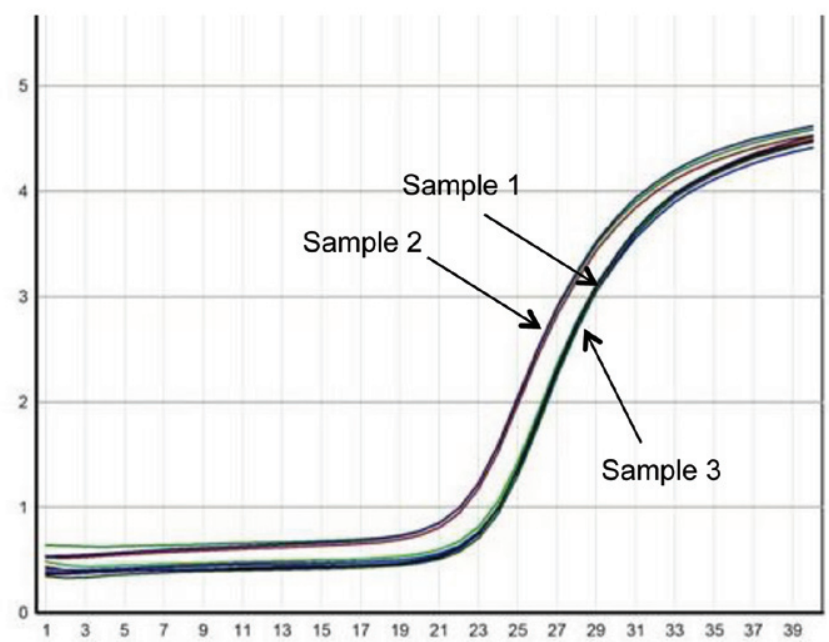

Figure 3. Molecular biology application of RNA isolated after pancreas perfusion. Three newborn pig pancreata were perfused with RNAlater. Isolated RNA (500 ng) was subjected to qRT-PCR for CFTR. B-actin was the housekeeping gene. Each sample was run in triplicate. The figure shows the amplification curve of the specimens. Sample $1 \Delta \mathrm{CT}$, -4.52 ; sample $2 \Delta \mathrm{CT}$, -3.99 ; sample 3 $\triangle \mathrm{CT},-4.89$. 

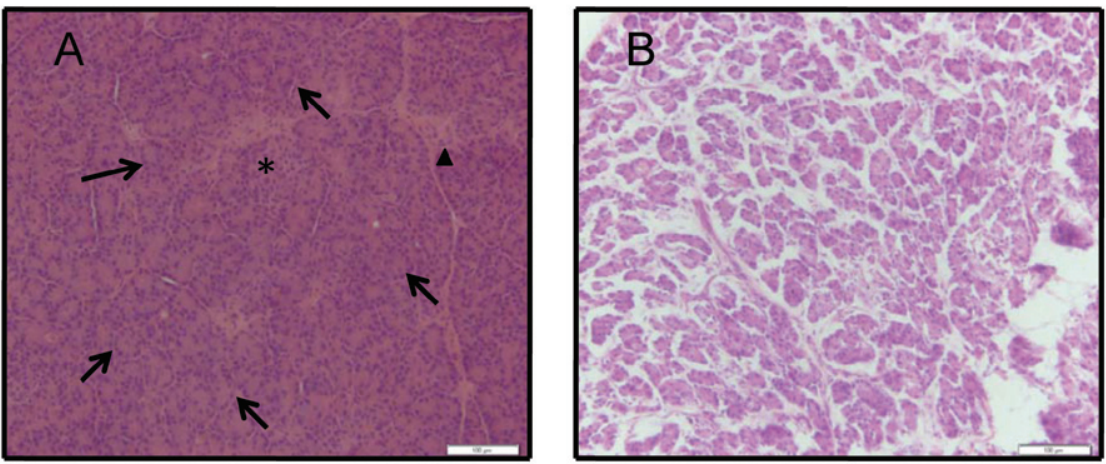

Figure 4. Duct perfusion with RNase inhibitor causes histological artifacts. Newborn pig pancreas histology from samples that were not perfused $(A)$ or processed after pancreatic duct was perfused with RNAlater (B). (A) Normal pancreatic architecture with acini (arrows), ducts (arrowhead), and islets $\left(^{*}\right)$. (B) Multiple white spaces created by the duct perfusion (artifacts) and overall distorted tissue architecture. Frozen sections of the pancreas $(10 \mu \mathrm{m})$ with hematoxylen \& eosin stain. Scale bar, $100 \mu \mathrm{m}$.

We applied the pancreas perfusion technique for RNA isolation to two different species, at two different age groups, newborn pigs and adult mice. Because the University of Iowa does not have the facilities to raise pigs to reach adulthood, we were not able to demonstrate whether our technique could be applicable to adult pigs.

In summary, our method is a simplified and more versatile modification of the in situ ductal perfusion technique reported by Mullin et al. (3). Our pancreas perfusion method does not interfere with the RNA quality and integrity that can be associated with tissue storage and performs as if RNA is isolated immediately without freezing. The technique yields excellent quality RNA from pancreas for molecular biology studies and does not create histological artifacts.

\section{Acknowledgments}

This work was supported by National institute of Health (NIH) DK084049 (A.U.). This paper is subject to the NIH Public Access Policy. We would like to thank Dr. Bahri Karacay for his input in preparing Figure 3.

\section{Competing interests}

The authors declare no competing interests.

\section{References}

1. Hembree, M.J., K. Prasadan, P. Manna, B. Preuett, T. Spilde, A. Bhatia, H. Kobayashi, B. Buckingham, et al. 2001. Semiquantitative polymerase chain reaction in RNase-producing tissues: Analysis of the developing pancreas. J. Pediatr. Surg. 36:1629-1632.

2. Kiba, T., Y. Kintaka, E. Nakada, Y. Suzuki, S. Inoue, and Y. Ishigaki. 2007. High-quality RNA extraction from rat pancreas for microarray analysis. Pancreas 35:98-100.

3. Mullin, A.E., G. Soukatcheva, C.B. Verchere, and J.K. Chantler. 2006. Application of in situ ductal perfusion to facilitate isolation of highquality RNA from mouse pancreas. BioTechniques 40:617-621.

4.Li, D., W. Ren, X. Wang, F. Wang, Y. Gao, Q. Ning, Y. Han, T. Song, and S. Lu. 2009. A modified method using TRIzol reagent and liquid nitrogen produces high-quality RNA from rat pancreas. Appl. Biochem. Biotechnol. 158:253-261.

5. Sparmann, G., A. Jaschke, M. Loehr, S. Liebe, and J. Emmrich. 1997. Tissue homogenization as a key step in extracting RNA from human and rat pancreatic tissue. BioTechniques 22:408-410, 412

6. Gill, S.S., R.A. Aubin, C.A. Bura, I.H. Curran, and T.I. Matula. 1996. Ensuring recovery of intact RNA from rat pancreas. Mol. Biotechnol. 6:359362.

7. Chirgwin, J.M., A.E. Przybyla, R.J. MacDonald, and W.J. Rutter. 1979. Isolation of biologically active ribonucleic acid from sources enriched in ribonuclease. Biochemistry 18:5294-5299.

8. Fleige, S. and M.W. Pfaffl. 2006. RNA integrity and the effect on the real-time qRT-PCR performance. Mol. Aspects Med. 27:126-139.

9. Chomczynski, P. and N. Sacchi. 1987. Single-step method of RNA isolation by acid guanidinium thiocyanate-phenol-chloroform extraction. Anal. Biochem. 162:156-159.

10. Rogers, C.S., Y. Hao, T. Rokhlina, M. Samuel, D.A. Stoltz, Y. Li, E. Petroff, D.W. Vermeer, et al. 2008. Production of CFTR-null and CFTRDeltaF508 heterozygous pigs by adeno-associated virus-mediated gene targeting and somatic cell nuclear transfer. J. Clin. Invest. 118:1571-1577.

11. Bova, G.S., I.A. Eltoum, J.A. Kiernan, G.P. Siegal, A.R. Frost, C.J. Best, J.W. Gillespie, G.H.Su, and M.R.Emmert-Buck. 2005. Optimal molecular profiling of tissue and tissue components: defining the best processing and microdissection methods for biomedical applications. Mol. Biotechnol. 29:119-152.

Received 22 September 2011; accepted 11 April 2012.

Address correspondence to Aliye Uc, 2865 JPP Pediatrics, University of Iowa, 200 Hawkins Drive, Iowa City, IA, USA.Email: aliye-uc@uiowa.edu

To purchase reprints of this article, contact: biotechniques@fosterprinting.com 